\title{
The beta Marshall-Olkin family of distributions
}

\author{
Morad Alizadeh ${ }^{1}$, Gauss M. Cordeiro ${ }^{2}$, Edleide de Brito ${ }^{3 *}$ and Clarice Garcia B. Demétrio ${ }^{4}$
}

*Correspondence:
edbnet@gmail.com
3Departamento de Estatística,
Universidade Federal da Bahia,
Salvador, Bahia, Brazil
Full list of author information is
available at the end of the article

available at the end of the article

\begin{abstract}
We study general mathematical properties of a new generator of continuous distributions with three extra shape parameters called the beta Marshall-Olkin family. We present some special models and investigate the asymptotes and shapes. The new density function can be expressed as a mixture of exponentiated densities based on the same baseline distribution. We derive a power series for its quantile function. Explicit expressions for the ordinary and incomplete moments, quantile and generating functions, Bonferroni and Lorenz curves, Shannon and Rényi entropies and order statistics, which hold for any baseline model, are determined. We discuss the estimation of the model parameters by maximum likelihood and illustrate the flexibility of the family by means of two applications to real data.
\end{abstract}

PACS: $02.50 . \mathrm{Ng}, 02.50 . \mathrm{CW}, 02.50 .-\mathrm{r}$

Mathematics Subject Classification (2010): 62E10, 60E05, 62 P99

Keywords: Generated family; Marshall-Olkin family; Maximum likelihood; Moment; Order statistic; Quantile function; Rényi entropy

\section{Introduction}

Recently, some attempts have been made to define new families to extend well-known distributions and at the same time provide great flexibility in modelling data in practice. So, several classes by adding one or more parameters to generate new distributions have been proposed in the statistical literature. Some well-known generators are: the MarshallOlkin generated (MO-G) by (Marshall and Olkin 1997), the beta-G by (Eugene et al. 2002), the Kumaraswamy-G (Kw-G for short) by (Cordeiro and Castro 2011), the McDonald-G (Mc-G) by (Alexander et al. 2012), the gamma-G by (Zografos and Balakrishnan 2009), the transformer (T-X) by (Alzaatreh et al. 2013), the Weibull-G by (Bourguignon et al. 2014) and the exponentiated half-logistic by (Cordeiro et al. 2014).

Let $r(t)$ be the probability density function (pdf) of a random variable $T \in[d, e]$ for $-\infty \leq d<e<\infty$ and let $W[G(x)]$ be a function of the cumulative distribution function (cdf) of a random variable $X$ such that $W[G(x)]$ satisfies the following conditions:

$\left\{\begin{array}{l}\text { (i) } W[G(x)] \in[d, e] \\ \text { (ii) } W[G(x)] \text { is differentiable and monotonically non-decreasing, and } \\ \text { (iii) } W[G(x)] \rightarrow d \text { as } x \rightarrow-\infty \text { and } W[G(x)] \rightarrow e \text { as } x \rightarrow \infty .\end{array}\right.$

(C) 2015 Alizadeh et al. This is an Open Access article distributed under the terms of the Creative Commons Attribution License (http://creativecommons.org/licenses/by/4.0), which permits unrestricted use, distribution, and reproduction in any medium, provided the original work is properly credited. 
Alzaatreh et al. 2013 defined the T-X family cdf by

$$
F(x)=\int_{d}^{W[G(x)]} r(t) d t,
$$

where $W[G(x)]$ satisfies the conditions (1). The pdf corresponding to (2) is given by

$$
f(x)=\left\{\frac{d}{d x} W[G(x)]\right\} r\{W[G(x)]\} .
$$

In this paper, we propose a new wider class of continuous distributions called the beta Marshall-Olkin (BMO) family by taking $W[G(x)]=\frac{G(x ; \xi)}{c+(1-c) G(x ; \xi)}$ and $r(t)=$ $\frac{1}{B(a, b)} t^{a-1}(1-t)^{b-1}, 0<t<1, a, b, c>0$. Its cdf is given by

$$
F(x ; a, b, c, \xi)=I_{\frac{G(x ; \xi)}{c+(1-c) G(x ; \xi)}}(a, b),
$$

where $I_{x}(a, b)=B(a, b)^{-1} \int_{0}^{x} t^{a-1}(1-t)^{b-1} d t$ denotes the incomplete beta function ratio, $G(x ; \xi)$ is the baseline cdf depending on a parameter vector $\xi$ and $a>0, b>0$ and $c>0$ are three additional shape parameters. For each baseline G, the BMO-G distribution is defined by the cdf (3). Equation (3) includes as special cases the beta-G, Marshall-OlkinG (MOG), exponentiated Marshal-Olkin-G (EMOG) and exponentiated classes as those listed in Table 1.

This paper is organized as follows. In Section 2, we provide a physical interpretation of the BMO-G family. Three special cases of this family are defined in Section 3. In Section 4, the shape of the density and hazard rate functions are described analytically. Some useful expansions are derived in Section 5. In Section 6, we obtain a power series for the BMO-G quantile function (qf). In Section 7, we propose explicit expressions for the ordinary and incomplete moments using the qf expansion. The generating function and mean deviations are derived in Sections 8 and 9, respectively. General expressions for the Rényi and Shannon entropies are presented in Section 10. The order statistics are investigated in Section 11. Estimation of the model parameters by maximum likelihood is performed in Section 12. Applications to two real data sets illustrate the performance of the new family in Section 13. The paper is concluded in Section 14.

\section{The new density}

The density function corresponding to (3) is given by

$$
f(x ; a, b, c, \xi)=\frac{c^{b} g(x ; \xi) G(x ; \xi)^{a-1} \bar{G}(x ; \xi)^{b-1}}{B(a, b)[c+(1-c) G(x ; \xi)]^{a+b}},
$$

where $g(x ; \xi)$ is the baseline pdf. Equation (4) will be most tractable when $G(x ; \xi)$ and $g(x ; \xi)$ have simple analytic expressions. Hereafter, a random variable $X$ with density

Table 1 Some special models

\begin{tabular}{lllll}
\hline$a$ & $b$ & $c$ & $G(x)$ & Reduced distributions \\
\hline- & - & 1 & $G(x)$ & Beta-G family (Eugene et al. 2002; Jones 2004) \\
1 & - & - & $G(x)$ & Generalized Marshal-Olkin family (Jayakumar and Mathew 2008) \\
1 & - & - & $G(x)$ & Exponentiated Marshall-Olkin family (New) \\
1 & 1 & - & $G(x)$ & Marshall-Olkin family (Marshall and Olkin 1997) \\
1 & - & 1 & $G(x)$ & Proportional hazard rate family (Gupta et al. 1998) \\
- & 1 & 1 & $G(x)$ & Proportional reversed hazard rate family (Gupta and Gupta 2007) \\
1 & 1 & 1 & $G(x)$ & $G(x)$ \\
\hline
\end{tabular}


function (4) is denoted by $X \sim \operatorname{BMO}-\mathrm{G}(a, b, c, \xi)$. Further, we can omit sometimes the dependence on the vector $\boldsymbol{\xi}$ of parameters and write simply $G(x)=G(x ; \xi)$.

The hazard rate function (hrf) of $X$ becomes

$$
\tau(x ; a, b, c, \boldsymbol{\xi})=\frac{c^{b} g(x ; \xi) G(x ; \xi)^{a-1} \bar{G}(x ; \xi)^{b-1}}{B(a, b)[c+(1-c) G(x ; \xi)]^{a+b}\left[1-I_{\frac{G(x ; \xi)}{c+(1-c) G(x ; \xi)}}(a, b)\right]}
$$

The BMO family is easily simulated by inverting (3) as follows: if $V$ has a beta distribution with positive parameters $a$ and $b$, the solution of the nonlinear equation

$$
x_{q}=G^{-1}\left\{\frac{c V}{1-(1-c) V} ; \xi\right\}
$$

has the density function (4).

The basic motivations for using the BMO family in practice are: (i) to make the kurtosis more flexible compared to the baseline model; (ii) to produce a skewness for symmetrical distributions; (iii) to construct heavy-tailed distributions that are not longer-tailed for modeling real data; (iv) to generate distributions with symmetric, left-skewed, rightskewed and reversed-J shaped; (v) to define special models with all types of the hrf; (vi) to generate a large number of special distributions as those presented in Table 1; and (vii) to provide consistently better fits than other generated models under the same baseline distribution. A simple example of (ii): the normal distribution is symmetric, but the beta Marshall-Olkin normal (BMO-N) becomes skewed. The fact (vii) is well-demonstrated by fitting the BMO-N and beta Marshall-Olkin Weibull (BMO-W) distributions to two real data sets in Section 13. However, we expect that there are other contexts in which the BMO special models can produce worse fits than other generated distributions. Clearly, the results in Section 13 indicate that the new family is a very competitive class to other known generators with at most three extra shape parameters.

\section{Some special BMO distributions}

The BMO-G density function (4) allows for greater flexibility of its tails and can be widely applied in many areas. The new family extends several widely-known distributions in the literature. Here, we present a few of its many special models.

\subsection{The BMO-N distribution}

The BMO-N pdf is obtained from (4) by taking the normal $N(\mu, \sigma)$ as the parent distribution, where $\xi=(\mu, \sigma)$. Then,

$$
f(x ; a, b, c, \alpha, \beta)=\frac{c^{b} \phi\left(\frac{x-\mu}{\sigma}\right)\left[\Phi\left(\frac{x-\mu}{\sigma}\right)\right]^{a-1}\left[1-\Phi\left(\frac{x-\mu}{\sigma}\right)\right]^{b-1}}{B(a, b)\left[c+(1-c) \Phi\left(\frac{x-\mu}{\sigma}\right)\right]^{a+b}},
$$

where $x \in R, \mu \in \boldsymbol{R}$ is a location parameter, $\sigma>0$ is a scale parameter, $\phi(\cdot)$ and $\Phi(\cdot)$ are the pdf and cdf of the standard normal distribution, respectively. The standard BMO-N density comes when $\mu=0$ and $\sigma=1$. For $a=b=c=1$, it reduces to the normal density. Plots of (6) for some parameter values are displayed in Fig. 1. 

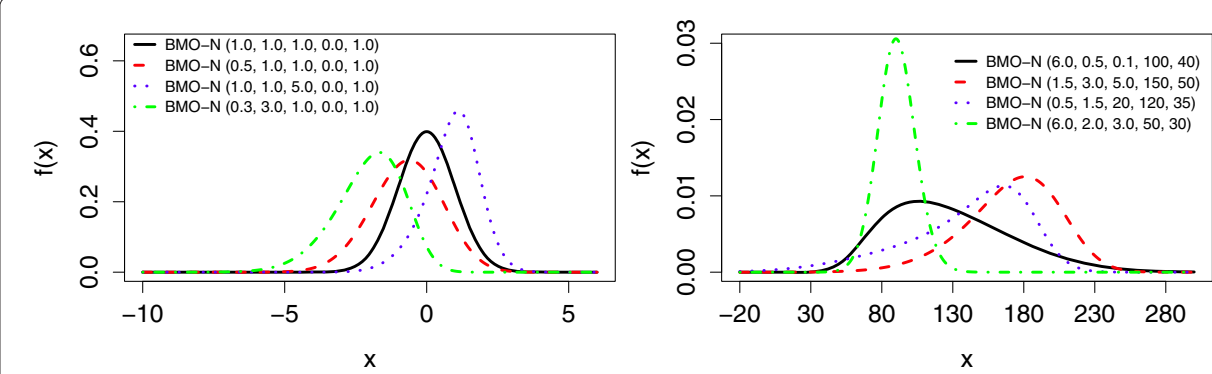

Fig. 1 The BMO-N density function for some parameter values

\subsection{The BMO-W distribution}

Let $G(x ; \xi)=1-\exp \left[-(\alpha x)^{\beta}\right]$ be the Weibull cdf with scale parameter $\alpha>0$ and shape parameter $\beta>0$, where $\xi=(\alpha, \beta)$. The BMO-W pdf (for $x>0$ ) reduces to

$$
f(x ; a, b, c, \alpha, \beta)=\frac{c^{b} \beta \alpha^{\beta} x^{\beta-1} \exp \left[-b(\alpha x)^{\beta}\right]\left[1-\exp \left[-(\alpha x)^{\beta}\right]\right]^{a-1}}{B(a, b)\left[c+(1-c)\left[1-\exp \left[-(\alpha x)^{\beta}\right]\right]\right]^{a+b}} .
$$

The Weibull pdf (with parameters $\alpha$ and $\beta$ ) is a special case for $a=b=c=1$. Some possible shapes of the BMO-W pdf and hrf are displayed in Fig. 2.

\subsection{The Beta Marshall-Olkin gamma (BMO-Ga) distribution}

The gamma cumulative distribution (for $x>0$ ) with shape parameter $\alpha>0$ and scale parameter $\beta>0, \xi=(\alpha, \beta)$, is given by

$$
G(x ; \xi)=\frac{\gamma(\alpha, x / \beta)}{\Gamma(\alpha)}=\gamma_{1}(\alpha, x / \beta),
$$

where $\Gamma(p)=\int_{0}^{\infty} w^{p-1} \mathrm{e}^{-w} d w$ is the gamma function and $\gamma(\alpha, z)=\int_{0}^{z} w^{\alpha-1} \mathrm{e}^{-w} d w$ is the incomplete gamma function. The BMO-Ga pdf (for $x>0$ ) becomes

$$
f(x ; a, b, \alpha, \beta)=\frac{c^{b} x^{a-1} \mathrm{e}^{-\mathrm{x} / \beta}\left[\gamma_{1}(\alpha, \mathrm{x} / \beta)\right]^{\mathrm{a}-1}\left[1-\gamma_{1}(\alpha, \mathrm{x} / \beta)\right]^{\mathrm{b}-1}}{\beta^{\alpha} \Gamma(\alpha) B(a, b)\left[c+(1-c) \gamma_{1}(\alpha, x / \beta)\right]^{a+b}} .
$$

For $c=1$, we obtain the beta Weibull (BW) distribution. The beta Marshall-Olkin exponential (BMO-E) distribution corresponds to $\beta=1$. Figure 3 displays some BMO-Ga pdf's and hrf's.
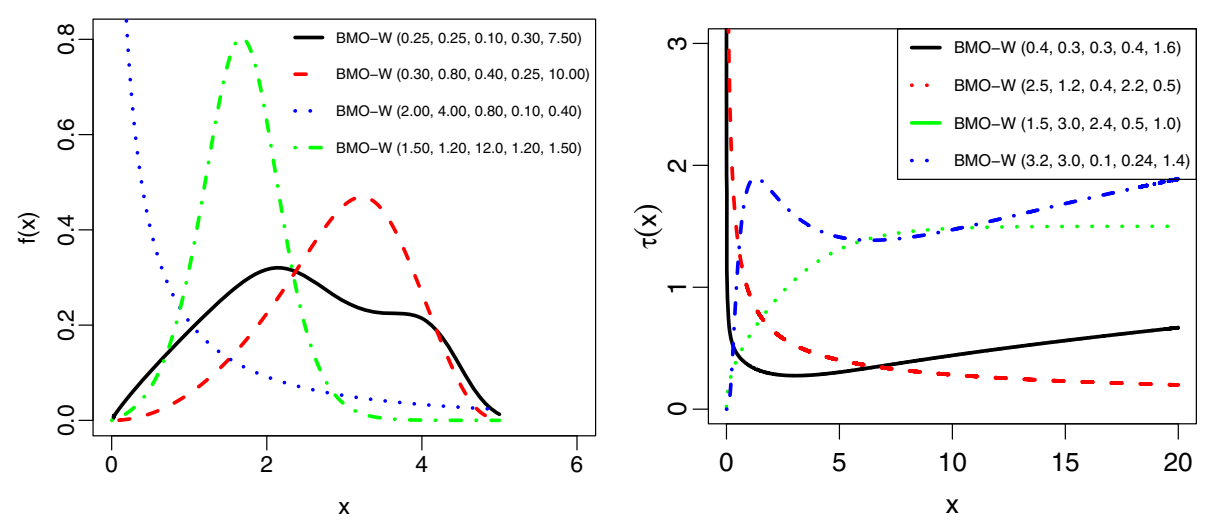

Fig. 2 The BMO-W density and hazard rate functions for some parameter values 

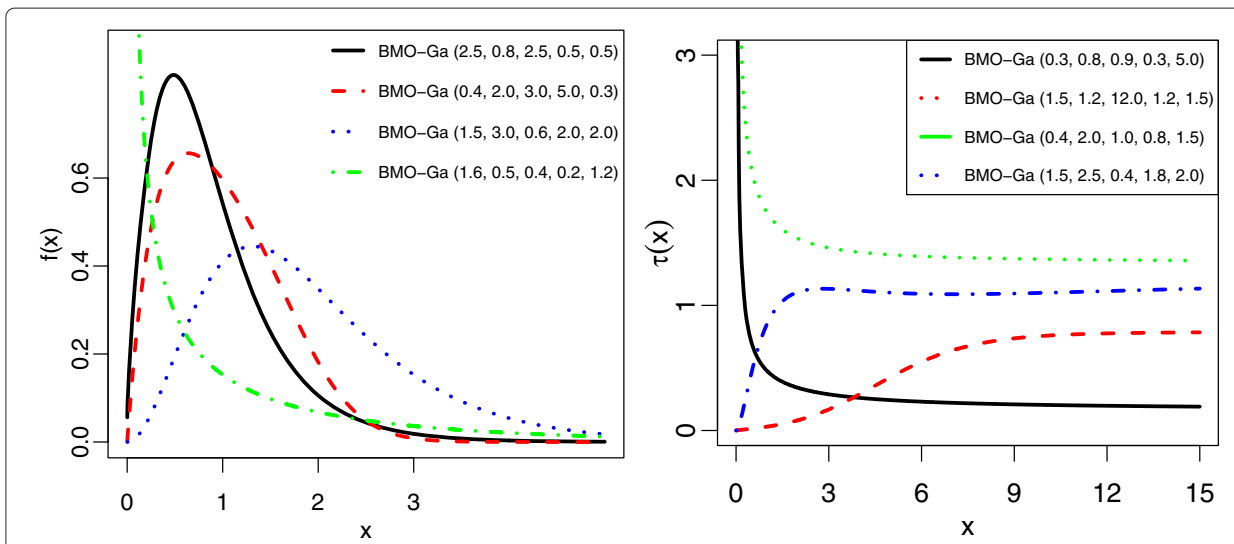

Fig. 3 The BMO-Ga density and hazard rate functions for some parameter values

\section{Asymptotics and shapes}

Corollary 1. The asymptotics of Eqs. (3), (4) and (5) as $G(x) \rightarrow 0$ are given by

$$
\begin{aligned}
& F(x) \sim \frac{G(x)^{a}}{a c^{a} B(a, b)} \text { as } \quad G(x) \rightarrow 0, \\
& f(x) \sim \frac{g(x) G(x)^{a-1}}{c^{a} B(a, b)} \text { as } G(x) \rightarrow 0, \\
& \tau(x) \sim \frac{g(x) G(x)^{a-1}}{c^{a} B(a, b)} \text { as } G(x) \rightarrow 0 .
\end{aligned}
$$

Corollary 2. The asymptotics of Eqs. (3), (4) and (5) as $x \rightarrow \infty$ are given by

$$
\begin{aligned}
& 1-F(x) \sim \frac{c^{b} \bar{G}(x)^{b}}{b B(a, b)} \text { as } x \rightarrow \infty, \\
& f(x) \sim \frac{c^{b} g(x) \bar{G}(x)^{b-1}}{B(a, b)} \text { as } x \rightarrow \infty, \\
& \tau(x) \sim \frac{b g(x)}{\bar{G}(x)} \text { as } x \rightarrow \infty .
\end{aligned}
$$

The shapes of the density and hazard rate functions can be described analytically. The critical points of the BMO-G density function are the roots of the equation:

$$
\frac{d \log [f(x)]}{d x}=\frac{g^{\prime}(x)}{g(x)}+\frac{(a-1) g(x)}{G(x)}+\frac{(1-b) g(x)}{\bar{G}(x)}+\frac{(c-1)(a+b) g(x)}{c+(1-c) G(x)} .
$$

There may be more than one root to (7). If $x=x_{0}$ is a root of (7) then it corresponds to a local maximum, a local minimum or a point of inflexion depending on whether $\lambda\left(x_{0}\right)<$ $0, \lambda\left(x_{0}\right)>0$ or $\lambda\left(x_{0}\right)=0$, where $\lambda(x)=\frac{d^{2} \log [f(x)]}{d x^{2}}$ is given by

$$
\begin{aligned}
\lambda(x)= & \frac{g^{\prime \prime}(x) g(x)-\left[g^{\prime}(x)\right]^{2}}{g(x)^{2}}+(a-1) \frac{g^{\prime}(x) G(x)-g(x)^{2}}{G(x)^{2}}+(1-b) \frac{g^{\prime}(x) \bar{G}(x)+g(x)^{2}}{\bar{G}(x)^{2}} \\
& +\frac{(c-1)(a+b) g^{\prime}(x)}{c+(1-c) G(x)}+\frac{(c-1)^{2}(a+b) g(x)^{2}}{[c+(1-c) G(x)]^{2}} .
\end{aligned}
$$


The critical points of $\tau(x)$ are the roots of the equation:

$$
\begin{aligned}
& \frac{d \log [\tau(x)]}{d x}=\frac{g^{\prime}(x)}{g(x)}+\frac{(a-1) g(x)}{G(x)}+\frac{(1-b) g(x)}{\bar{G}(x)}+\frac{(c-1)(a+b) g(x)}{c+(1-c) G(x)} \\
& -\frac{c^{b} g(x) G(x)^{a-1} \bar{G}(x)^{b-1}}{B(a, b)[c+(1-c) G(x)]^{a+b}\left[1-I_{\overline{c+(1-c) G(x)}}(a, b)\right]} .
\end{aligned}
$$

There may be more than one root to (8). If $x=x_{0}$ is a root of (8) then it corresponds to a local maximum, a local minimum or a point of inflexion depending on whether $\varsigma\left(x_{0}\right)<$ $0, \varsigma\left(x_{0}\right)>0$ or $\varsigma\left(x_{0}\right)=0$, where $\varsigma(x)=\frac{d^{2} \log [\tau(x)]}{d x^{2}}$ is given by

$$
\begin{aligned}
& \varsigma(x)=\frac{g^{\prime \prime}(x) g(x)-\left[g^{\prime}(x)\right]^{2}}{g(x)^{2}}+(a-1) \frac{g^{\prime}(x) G(x)-g(x)^{2}}{G(x)^{2}}+(1-b) \frac{g^{\prime}(x) \bar{G}(x)+g(x)^{2}}{\bar{G}(x)^{2}} \\
& +\frac{(c-1)(a+b) g^{\prime}(x)}{c+(1-c) G(x)}+\frac{(c-1)^{2}(a+b) g(x)^{2}}{[c+(1-c) G(x)]^{2}} \\
& +\frac{c^{b} g^{\prime}(x) G(x)^{a-1} \bar{G}(x)^{b-1}}{B(a, b)[c+(1-c) G(x)]^{a+b}\left[1-I_{\frac{G(x)}{c+(1-c) G(x)}}(a, b)\right]} \\
& +\frac{c^{b}(a-1) g(x)^{2} G(x)^{a-2} \bar{G}(x)^{b-1}}{B(a, b)[c+(1-c) G(x)]^{a+b}\left[1-I_{\frac{G(x)}{c+(1-c) G(x)}}(a, b)\right]} \\
& -\frac{c^{b}(b-1) g(x)^{2} G(x)^{a-1} \bar{G}(x)^{b-2}}{B(a, b)[c+(1-c) G(x)]^{a+b}\left[1-I_{\frac{G(x)}{c+(1-c) G(x)}}(a, b)\right]} \\
& +\left\{\frac{c^{b} g(x) G(x)^{a-1} \bar{G}(x)^{b-1}}{B(a, b)[c+(1-c) G(x)]^{a+b}\left[1-I_{\frac{G(x)}{c+(1-c) G(x)}}(a, b)\right]}\right\}^{2} .
\end{aligned}
$$

\section{Useful representation}

By using the generalized binomial expansion, we can prove that the cdf (3) of $X$ admits the expansion

$$
F(x ; a, b, c, \xi)=\sum_{i, j, l=0}^{\infty} \sum_{k=0}^{l} \frac{(-1)^{i+l+k}(1-c)^{i}\left(\begin{array}{c}
b-1 \\
i
\end{array}\right)\left(\begin{array}{c}
-a-i \\
j
\end{array}\right)\left(\begin{array}{c}
a+i+j \\
l
\end{array}\right)\left(\begin{array}{l}
l \\
k
\end{array}\right)}{c^{a+i+j} B(a, b)(a+i)} G(x)^{k} .
$$

By exchanging the indices $l$ and $k$ in the sum symbol, we can write

$$
F(x ; a, b, c, \xi)=\sum_{i, j, k=0}^{\infty} \sum_{l=k}^{\infty} \frac{(-1)^{i+l+k}(1-c)^{i}\left(\begin{array}{c}
b-1 \\
i
\end{array}\right)\left(\begin{array}{c}
-a-i \\
j
\end{array}\right)\left(\begin{array}{c}
a+i+j \\
l
\end{array}\right)\left(\begin{array}{l}
l \\
k
\end{array}\right)}{c^{a+i+j} B(a, b)(a+i)} G(x)^{k},
$$

and then

$$
F(x ; a, b, c, \xi)=\sum_{k=0}^{\infty} \beta_{k} G(x)^{k},
$$

where (for $k \geq 0$ )

$$
\beta_{k}=\sum_{i, j=0}^{\infty} \sum_{l=k}^{\infty} \frac{(-1)^{i+l+k}(1-c)^{i}\left(\begin{array}{c}
b-1 \\
i
\end{array}\right)\left(\begin{array}{c}
-a-i \\
j
\end{array}\right)\left(\begin{array}{c}
a+i+j \\
l
\end{array}\right)\left(\begin{array}{l}
l \\
k
\end{array}\right)}{c^{a+i+j} B(a, b)(a+i)} .
$$


The density function of $X$ can be expressed as a mixture of exp-G densities

$$
f(x ; a, b, c, \xi)=\sum_{k=0}^{\infty} \beta_{k+1} h_{k+1}(x),
$$

where $h_{k+1}(x)=(k+1) g(x ; \xi) G^{k}(x ; \xi)$ denotes the exp-G density function with power parameter $k+1$.

Thus, some mathematical properties of the new model can be derived from those exp-G properties. For example, the ordinary and incomplete moments and moment generating function (mgf) of $X$ can be obtained from those quantities of the exp-G distribution.

The formulae derived throughout the paper can be easily handled in most symbolic computation software platforms such as Maple, Mathematica and Matlab. These platforms allow to deal with analytic expressions of formidable size and complexity. Established explicit expressions to calculate statistical measures can be more efficient than computing them directly by numerical integration. The infinity limit in these sums can be substituted by a large positive integer such as 20 or 30 for most practical purposes.

\section{Quantile power series}

The qf of $X$, say $x=Q(u)=F^{-1}(u)$, can be obtained by inverting (3). Let $z=Q_{a, b}(u)$ be the beta qf. Then,

$$
x=Q(u)=Q_{G}\left\{\frac{c Q_{a, b}(u)}{1-(1-c) Q_{a, b}(u)}\right\} .
$$

It is possible to obtain some expansions for $Q_{a, b}(u)$ in the wolfram website ${ }^{1}$ such as

$$
z=Q_{a, b}(u)=\sum_{i=0}^{\infty} e_{i} u^{i / a}
$$

where $e_{i}=[a B(a, b)]^{1 / a} d_{i}$ and $d_{0}=0, d_{1}=1, d_{2}=(b-1) /(a+1)$,

$$
\begin{aligned}
d_{3}= & \frac{(b-1)\left(a^{2}+3 a b-a+5 b-4\right)}{2(a+1)^{2}(a+2)}, \\
d_{4} & =(b-1)\left[a^{4}+(6 b-1) a^{3}+(b+2)(8 b-5) a^{2}+\left(33 b^{2}-30 b+4\right) a\right. \\
& +b(31 b-47)+18] /\left[3(a+1)^{3}(a+2)(a+3)\right], \ldots
\end{aligned}
$$

The effects of the shape parameters $a, b$ and $c$ on the skewness and kurtosis of $X$ can be based on quantile measures. The shortcomings of the classical kurtosis measure are wellknown. The Bowley skewness (Kenney and Keeping 1962) is one of the earliest skewness measures defined by the average of the quartiles minus the median, divided by half the interquartile range, namely

$$
B=\frac{Q\left(\frac{3}{4}\right)+Q\left(\frac{1}{4}\right)-2 Q\left(\frac{1}{2}\right)}{Q\left(\frac{3}{4}\right)-Q\left(\frac{1}{4}\right)} .
$$

Since only the middle two quartiles are considered and the outer two quartiles are ignored, this adds robustness to the measure. The Moors kurtosis (Moors 1988) is based on octiles

$$
M=\frac{Q\left(\frac{3}{8}\right)-Q\left(\frac{1}{8}\right)+Q\left(\frac{7}{8}\right)-Q\left(\frac{5}{8}\right)}{Q\left(\frac{6}{8}\right)-Q\left(\frac{2}{8}\right)} .
$$

These measures are less sensitive to outliers and they exist even for distributions without moments. 


\section{Moments}

We assume that $Y$ is a random variable having the baseline $\operatorname{cdf} G(x)$. The moments of $X$ can be determined from the $(r, k)$ th probability weighted moment (PWM) of $Y$ defined by

$$
\omega_{r, k}=\mathrm{E}\left[Y^{r} G(Y)^{k}\right]=\int_{-\infty}^{\infty} x^{r} G(x)^{k} g(x) d x .
$$

The PWMs are used to derive estimators of the parameters and quantiles of generalized distributions. The moment method of estimation is formulated by equating the population and sample PWMs. These moments have low variance and no severe biases, and they compare favorably with estimators obtained by maximum likelihood. However, the maximum likelihood method is adopted in Section 12 since it is easier to estimate the BMO-G parameters because of several computer routines available in widely known softwares. The maximum likelihood estimators (MLEs) enjoy desirable properties and can be used for constructing confidence intervals and also for test statistics.

We can write from Eq. 10

$$
\mu_{r}^{\prime}=\mathrm{E}\left(X^{r}\right)=\sum_{k=0}^{\infty}(k+1) \beta_{k+1} \omega_{r, k}
$$

where $\omega_{r, k}=\int_{0}^{1} Q_{G}(u)^{r} u^{k} d u$ can be computed at least numerically from any baseline qf.

Thus, the moments of any BMO-G distribution can be expressed as an infinite weighted sum of the baseline PWMs. We now provide the PWMs for three distributions discussed in Section 3. For the BMO-N and BMO-Ga distributions introduced in Sections 3.1 and 3.3 , the quantities $\omega_{r, k}$ can be expressed in terms of the Lauricella functions of type A (see Exton 1978; Trott 2006) defined by

$$
\begin{aligned}
& F_{A}^{(n)}\left(a ; b_{1}, \ldots, b_{n} ; c_{1}, \ldots, c_{n} ; x_{1}, \ldots, x_{n}\right)= \\
& \sum_{m 1=0}^{\infty} \ldots \sum_{m_{n}=0}^{\infty} \frac{(a)_{m_{1}+\ldots+m_{n}}\left(b_{1}\right)_{m_{1}} \ldots\left(b_{n}\right)_{m_{n}}}{\left(c_{1}\right)_{m_{1}} \ldots\left(c_{n}\right)_{m_{n}}} \frac{x_{1}^{m_{1}} \ldots x_{n}^{m_{n}}}{m_{1} ! \ldots m_{n} !},
\end{aligned}
$$

where $(a)_{i}=a(a+1) \ldots(a+i-1)$ is the ascending factorial (with the convention that $\left.(a)_{0}=1\right)$.

In fact, (Cordeiro and Nadarajah 2011) determined $\omega_{r, k}$ for the standard normal distribution as

$$
\begin{aligned}
\omega_{r, k}= & 2^{r / 2} \pi^{-(k+1 / 2)} \sum_{\begin{array}{c}
l=0 \\
(r+k-l) \text { even }
\end{array}}^{k}\left(\begin{array}{l}
k \\
l
\end{array}\right) 2^{-l} \pi^{l} \Gamma\left(\frac{r+k-l+1}{2}\right) \\
& \times F_{A}^{(k-l)}\left(\frac{r+k-l+1}{2} ; \frac{1}{2}, \ldots, \frac{1}{2} ; \frac{3}{2}, \ldots, \frac{3}{2} ;-1, \ldots,-1\right) .
\end{aligned}
$$

This equation holds when $r+k-l$ is even and it vanishes when $r+k-l$ is odd. So, any BMO-N moment can be expressed as an infinite weighted linear combination of Lauricella functions of type A.

For the gamma distribution, the quantities $\omega_{r, k}$ can be expressed from Eq. (9) of (Cordeiro and Nadarajah 2011) as

$$
\omega_{r, k}=\frac{\Gamma(r+(k+1) \alpha)}{\alpha^{k} \beta^{r} \Gamma(\alpha)^{k+1}} F_{A}^{(k)}(r+(k+1) \alpha ; \alpha, \ldots, \alpha ; \alpha+1, \ldots, \alpha+1,-1, \ldots,-1) .
$$


As the last example, for the BMO-W distribution discussed in Section 3.2, the quantities $\omega_{r, k}$ reduce to

$$
\omega_{r, k}=\frac{\Gamma(r / \beta+1)}{\alpha^{r / \beta}} \sum_{s=0}^{k} \frac{(-1)^{s}}{(s+1)^{r / \beta+1}}\left(\begin{array}{l}
k \\
s
\end{array}\right) .
$$

Some important questions in economics are answered by knowing the mean and the shape of a distribution. Incomplete moments of an income distribution form natural building blocks for measuring inequality: for example, the Lorenz and Bonferroni curves depend upon the incomplete moments of the income distribution.

The $n$th incomplete moment of $X$ is defined by $m_{n}(y)=\int_{-\infty}^{y} x^{r} f(x) d x$. So, $m_{n}(y)$ follows as

$$
m_{n}(y)=\sum_{k=0}^{\infty} \beta_{k+1} \int_{0}^{G(y ; \xi)} Q_{G}(u)^{n} u^{k} d u .
$$

The integral in (13) can be computed at least numerically for most baseline distributions.

\section{Generating function}

We provide two formulae for the $\operatorname{mgf} M(s)=\mathrm{E}\left(\mathrm{e}^{s X}\right)$ of $X$. The first formula for $M(s)$ comes from Eq. (10) as

$$
M(s)=\sum_{k=0}^{\infty} \beta_{k+1} M_{k+1}(s),
$$

where $M_{k+1}(s)$ is the exp-G generating function with power parameter $k+1$.

The second formula for $M(s)$ follows in terms of the baseline qf as

$$
M(s)=\sum_{k=0}^{\infty}(k+1) \beta_{k+1} \rho_{k}(s),
$$

where the quantity $\rho_{k}(s)=\int_{0}^{1} \exp \left[s Q_{G}(u)\right] u^{k} d u$ can be computed numerically. Equations (14) and (15) are the main results of this section.

\section{Mean deviations}

The mean deviations about the mean $\left(\delta_{1}=\mathrm{E}\left(\left|X-\mu_{1}^{\prime}\right|\right)\right)$ and about the median $\left(\delta_{2}=\right.$ $\mathrm{E}(|X-M|))$ of $X$ can be expressed as

$$
\delta_{1}=2 \mu_{1}^{\prime} F\left(\mu_{1}^{\prime}\right)-2 m_{1}\left(\mu_{1}^{\prime}\right) \quad \text { and } \quad \delta_{2}=\mu_{1}^{\prime}-2 m_{1}(M),
$$

respectively, where $M=Q(0.5)$ is the median of $X, \mu_{1}^{\prime}=\mathrm{E}(X)$ comes from Eq. (12), $F\left(\mu_{1}^{\prime}\right)$ is easily calculated from Eq. (3) and $m_{1}(z)=\int_{-\infty}^{z} x f(x) d x$ is the first incomplete moment.

Now, we provide two alternative ways to compute $\delta_{1}$ and $\delta_{2}$. A general equation for $m_{1}(z)$ can be derived from Eq. (10) as

$$
m_{1}(z)=\sum_{k=0}^{\infty} \beta_{k+1} J_{k+1}(z),
$$

where $J_{k+1}(z)=\int_{-\infty}^{z} x h_{k+1}(x) d x$.

Equation (17) is the basic quantity to compute the mean deviations in Eq. 16. A simple application of it refers to the BMO-W model (Section 3.2). The exponentiated Weibull 
density function (for $x>0$ ) with power parameter $k+1$, shape parameter $\alpha$ and scale parameter $\beta$, is given by

$$
h_{k+1}(x)=(k+1) \alpha \beta^{\alpha} x^{\alpha-1} \exp \left\{-(\beta x)^{\alpha}\right\}\left[1-\exp \left\{-(\beta x)^{\alpha}\right\}\right]^{k},
$$

and then

$$
J_{k+1}(z)=\alpha(k+1) \beta^{\alpha} \sum_{r=0}^{\infty}(-1)^{r}\left(\begin{array}{l}
k \\
r
\end{array}\right) \int_{0}^{z} x^{\alpha} \exp \left\{-(r+1)(\beta x)^{\alpha}\right\} d x .
$$

Using the incomplete gamma function, the last integral reduces to

$$
J_{k+1}(z)=\beta^{-1} \sum_{r=0}^{\infty} \frac{(-1)^{r}(k+1)\left(\begin{array}{l}
k \\
r
\end{array}\right)}{(r+1)^{1+\alpha^{-1}}} \gamma\left(1+\alpha^{-1},(r+1)(\beta z)^{\alpha}\right) .
$$

A second general formula for $m_{1}(z)$ can be derived by setting $u=G(x)$ in Eq. 17

$$
m_{1}(z)=\sum_{k=0}^{\infty}(k+1) \beta_{k+1} T_{k}(z)
$$

where $T_{k}(z)=\int_{0}^{G(z)} Q_{G}(u) u^{k} d u$.

The main application of the first incomplete moment refers to the Bonferroni and Lorenz curves that are very useful in economics, reliability, demography, insurance and medicine. For a given probability $\pi$, applications of these equations can be addressed to obtain these curves defined by $B(\pi)=m_{1}(q) /\left(\pi \mu_{1}^{\prime}\right)$ and $L(\pi)=m_{1}(q) / \mu_{1}^{\prime}$, respectively, where $q=$ $Q(\pi)$ is calculated from the parent qf in (11). In Fig. 4, we plot the measures $B$ and $L$ of the BMO-N and BMO-W distributions. The plots indicate the variability of these measures on the shape parameters.
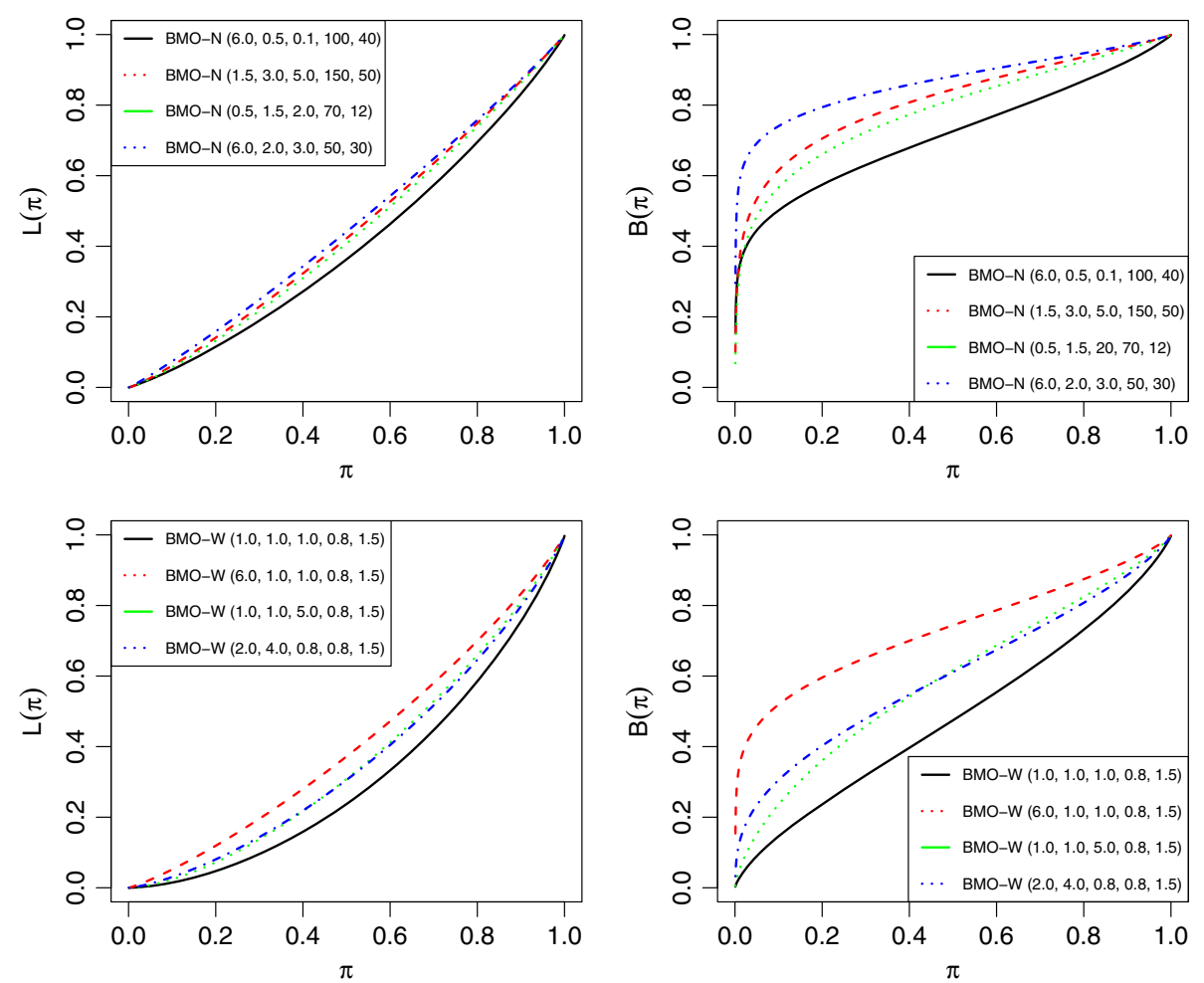

Fig. 4 Lorenz and Bonferroni curves for selected parameter values of the BMON and BMOW distributions 


\section{Entropies}

An entropy is a measure of variation or uncertainty of a random variable $X$. Two popular entropy measures are the Rényi and Shannon entropies (Rényi 1961; Shannon 1951). The Rényi entropy of a random variable with $\operatorname{pdf} f(x)$ is defined by

$$
I_{R}(c)=\frac{1}{1-\gamma} \log \left(\int_{0}^{\infty} f^{\gamma}(x) d x\right),
$$

for $\gamma>0$ and $\gamma \neq 1$. The Shannon entropy of a random variable $X$ is defined by $E\{-\log [f(X)]\}$. It is the special case of the Rényi entropy when $\gamma \uparrow 1$. Direct calculation yields

$$
\begin{aligned}
\mathrm{E}\{-\log [f(X)]\} & =-\log \left[\frac{c^{b}}{B(a, b)}\right]-\mathrm{E}\{\log [g(X ; \xi)]\}+(1-a) \mathrm{E}\{\log [G(x ; \xi)]\} \\
& +(1-b) \mathrm{E}\{\log [\bar{G}(x ; \xi)]\}+(a+b) \mathrm{E}\{\log [c+(1-c) G(x ; \xi)]\} .
\end{aligned}
$$

First, let

$$
A\left(a_{1}, a_{2}, a_{3} ; c\right)=\int_{0}^{1} \frac{u^{a_{1}}(1-u)^{a_{2}}}{[c+(1-c) u]^{a_{3}}} d u .
$$

Using the generalized binomial expansion, we obtain

$$
A\left(a_{1}, a_{2}, a_{3} ; c\right)=\sum_{i=0}^{\infty}(1-c)^{i} c^{-a_{3}-i}\left(\begin{array}{c}
-a_{3} \\
i
\end{array}\right) B\left(a_{1}+i+1, a_{2}+1\right) .
$$

After some algebraic manipulations, we have the following proposition.

Proposition 1. Let $X$ be a random variable with $p d f(4)$. Then,

$$
\begin{aligned}
& E\{\log [G(X ; \xi)]\}=\left.\frac{c^{b}}{B(a, b)} \frac{\partial}{\partial t} A(a+t-1, b-1, a+b ; c)\right|_{t=0} \\
& E\{\log [\bar{G}(X ; \xi)]\}=\left.\frac{c^{b}}{B(a, b)} \frac{\partial}{\partial t} A(a-1, b+t-1, a+b ; c)\right|_{t=0} \\
& E\{\log [c+(1-c) G(X ; \xi)]\}=\left.\frac{c^{b}}{B(a, b)} \frac{\partial}{\partial t} A(a-1, b-1, a+b-t ; c)\right|_{t=0} .
\end{aligned}
$$

The simplest formula for the entropy of $X$ is given by

$$
\begin{aligned}
\mathrm{E}\{-\log [f(X)]\} & =-\log [\alpha \lambda(1-p)]-\mathrm{E}\{\log [g(X ; \xi)]\} \\
& +\left.\frac{(1-a) c^{b}}{B(a, b)} \frac{\partial}{\partial t} A(a+t-1, b-1, a+b ; c)\right|_{t=0} \\
& +\left.\frac{(1-b) c^{b}}{B(a, b)} \frac{\partial}{\partial t} A(a-1, b+t-1, a+b ; c)\right|_{t=0} \\
& +\left.\frac{(a+b) c^{b}}{B(a, b)} \frac{\partial}{\partial t} A(a-1, b-1, a+b-t ; c)\right|_{t=0}
\end{aligned}
$$

After some algebra, we obtain an alternative expression for $I_{R}(\gamma)$

$$
I_{R}(\gamma)=\frac{\gamma}{1-\gamma} \log \left[\frac{c^{b}}{B(a, b)}\right]+\frac{1}{1-\gamma} \log \left\{\sum_{i, j=0}^{\infty} w_{i, j}^{\star} I(\gamma, a, j)\right\},
$$


where

$$
w_{i, j}^{\star}=(-1)^{j}(1-c)^{i} c^{-\gamma(a+b)-i}\left(\begin{array}{c}
-\gamma(a+b) \\
i
\end{array}\right)\left(\begin{array}{c}
\gamma(b-1) \\
j
\end{array}\right)
$$

and $I(\gamma, a, j)=\int_{0}^{\infty} g(x)^{\gamma} G(x)^{\gamma(a-1)+j}$

\section{Order statistics}

Order statistics make their appearance in many areas of statistical theory and practice. Suppose $X_{1}, \ldots, X_{n}$ is a random sample from any BMO-G distribution. Let $X_{i: n}$ denote the $i$ th order statistic. The pdf of $X_{i: n}$ can be expressed as

$$
f_{i: n}(x)=K f(x) F^{i-1}(x)\{1-F(x)\}^{n-i}=K \sum_{j=0}^{n-i}(-1)^{j}\left(\begin{array}{c}
n-i \\
j
\end{array}\right) f(x) F(x)^{j+i-1},
$$

where $K=n ! /[(i-1) !(n-i) !]$.

We can demonstrate that the density function of the $i$ th order statistic of any BMO-G distribution can be expressed as

$$
f_{i: n}(x)=\sum_{r, k=0}^{\infty} m_{r, k} h_{r+k+1}(x),
$$

where $h_{r+k+1}(x)$ denotes the exp-G density function with parameter $r+k+1$,

$$
m_{r, k}=\frac{n !(r+1)(i-1) ! \beta_{r+1}}{(r+k+1)} \sum_{j=0}^{n-i} \frac{(-1)^{j} f_{j+i-1, k}}{(n-i-j) !}
$$

$\beta_{r}$ is given by (9) and the quantities $f_{j+i-1, k}$ can be determined by $f_{j+i-1,0}=\beta_{0}^{j+i-1}$ and recursively (for $k \geq 1$ )

$$
f_{j+i-1, k}=\left(k \beta_{0}\right)^{-1} \sum_{m=1}^{k}[m(j+i)-k] \beta_{m} f_{j+i-1, k-m} .
$$

We can obtain the ordinary and incomplete moments, generating function and mean deviations of the BMO-G order statistics from Eq. (18) and some properties of the exp-G model.

\section{Estimation}

Here, we determine the MLEs of the model parameters of the new family from complete samples only. Let $x_{1}, \ldots, x_{n}$ be observed values from the BMO-G distribution with parameters $a, b, c$ and $\xi$. Let $\Theta=(a, b, c, \xi)^{\top}$ be the $r \times 1$ parameter vector. The total $\log$-likelihood function for $\Theta$ is given by

$$
\begin{aligned}
\ell & =\ell(\Theta)=n b \log (c)-n \log [B(a, b)]+\sum_{i=1}^{n} \log \left[g\left(x_{i}, \xi\right)\right] \\
& +(a-1) \sum_{i=1}^{n} \log \left\{G\left(x_{i}, \xi\right)\right\}+(b-1) \sum_{i=1}^{n} \log \left[\bar{G}\left(x_{i}, \xi\right)\right] \\
& -(a+b) \sum_{i=1}^{n} \log \left[c+(1-c) G\left(x_{i}, \xi\right)\right]
\end{aligned}
$$


Numerical maximization of (19) can be performed by using the RS method (Rigby and Stasinopoulos 2005) which is available in the gamlss package ( $R$ Development Core Team 2013), SAS (Proc NLMixed) or the Ox program (sub-routine MaxBFGS) (see Doornik 2007) or by solving the nonlinear likelihood equations obtained by differentiating (19). Let $U_{n}(\Theta)=\left(\partial \ell_{n} / \partial a, \partial \ell_{n} / \partial b, \partial \ell_{n} / \partial c, \partial \ell_{n} / \partial \xi\right)^{\top}$ be the score function, whose components are

$$
\begin{aligned}
U_{a}= & \frac{\partial \ell}{\partial a}=-n \psi(a)+n \psi(a+b)+\sum_{i=1}^{n} \log \left\{G\left(x_{i}, \xi\right)\right\}-\sum_{i=1}^{n} \log \left[c+(1-c) G\left(x_{i}, \xi\right)\right] \\
U_{b}= & \frac{\partial \ell}{\partial b}=n \log (c)-n \psi(b)+n \psi(a+b)+\sum_{i=1}^{n} \log \left\{\bar{G}\left(x_{i}, \xi\right)\right\} \\
& -\sum_{i=1}^{n} \log \left[c+(1-c) G\left(x_{i}, \xi\right)\right] \\
U_{c}= & \frac{\partial \ell}{\partial c}=\frac{n b}{c}-(a+b) \sum_{i=1}^{n} \frac{\bar{G}\left(x_{i}, \xi\right)}{c+(1-c) G\left(x_{i}, \xi\right)}
\end{aligned}
$$

and

$$
\begin{aligned}
U_{\xi}= & \frac{\partial \ell}{\partial \xi}=\sum_{i=1}^{n} \frac{g^{(\xi)}(x ; \xi)}{g(x ; \xi)}+(a-1) \sum_{i=1}^{n} \frac{G^{(\xi)}(x ; \xi)}{G(x ; \xi)}+(1-b) \sum_{i=1}^{n} \frac{G^{(\xi)}(x ; \xi)}{\bar{G}(x ; \xi)} \\
& +(c-1)(a+b) \sum_{i=1}^{n} \frac{G^{(\xi)}(x ; \xi)}{c+(1-c) G(x ; \xi)},
\end{aligned}
$$

where $h^{(\xi)}(\cdot)$ means the derivative of the function $h$ with respect to $\xi$. Setting these equations to zero, $U_{a}=U_{b}=U_{c}=U_{\xi}=\mathbf{0}$, and solving them simultaneously yields the MLE $\widehat{\Theta}$ of $\Theta$.

For interval estimation on the model parameters, it is required the observed information matrix, whose elements $U_{r s}=\partial^{2} \ell / \partial r \partial s$ (for $r, s=a, b, c, \xi$ ) can be computed numerically. Under standard regularity conditions (Cox and Hinkley 1979), we can approximate the distribution of $(\widehat{\Theta}-\Theta)$ by the multivariate normal $N_{r+3}\left(0, J(\Theta)^{-1}\right)$ distribution, where $r$ is the number of parameters of the baseline distribution.

We can compute the maximum values of the unrestricted and restricted log-likelihoods to construct likelihood ratio (LR) statistics for testing some sub-models of the BMO-G distribution. For example, we may use LR statistics to check if the fit using the BMO-W distribution is statistically "superior" to the fits using the BW, MOW, EW, EE and Weibull distributions for a given data set.

Often with lifetime data and reliability studies, one encounters censoring. Suppose that the lifetimes are independently distributed, and also independent from the censoring mechanism and censoring is random and noninformative. Considering right-censored lifetime data, we observe $x_{i}=\min \left(X_{i}, C_{i}\right)$ and $\delta_{i}=I\left(X_{i} \leq C_{i}\right)$ such that $\delta_{i}=1$ if $X_{i}$ is a time to event and $\delta_{i}=0$ if it is right censored for $i=1, \ldots, n$ where $X_{i}$ is the lifetime for the $i$ th individual and $C_{i}$ is the censoring for the $i$ th individual, $i=1, \ldots, n$. The censored likelihood $L(\Theta)$ for the model parameters is

$$
L(\Theta) \propto \prod_{i=1}^{n}\left[f\left(x_{i} ; a, b, c, \xi\right)\right]^{\delta_{i}}\left[S\left(x_{i} ; a, b, c, \xi\right)\right]^{1-\delta_{i}},
$$


where $S(x ; a, b, c, \xi)=1-F(x ; a, b, c, \xi)$ is the survival function obtained from (3) and $f(x ; a, b, c, \xi)$ is given by (4). We maximize the likelihood (20) in the same way as described before.

\section{Empirical illustration}

We illustrate the flexibility of the BMO-W and BMO-N distributions by means of two real data sets. Similar investigations could be performed for other BMO distributions. We have chosen these distributions because of the popularity of their baseline distributions. The computations are performed using the software $\mathrm{R}$ version 3.0.0 (package bbmle). The maximization follows the BFGS method with analytical derivatives. The algorithm used to estimate the model parameters converged for all current models.

\subsection{Illustration 1: Failure time data}

We next consider the data studied by (Murthy et al. 2004), which represent failure times for a particular windshield device. The windshield on a large aircraft is a complex piece of equipment, comprised basically of several layers of material, including a very strong outer skin with a heated layer just beneath it, all laminated under high temperature and pressure. Failures of these items are not structural failures. Instead, they typically involve damage or delamination of the nonstructural outer ply or failure of the heating system. These failures do not result in damage to the aircraft but do result in replacement of the windshield. We compare the results of the fits of the BMO-W distribution, its special models (W, EW, BW, MOW and EMOW) and the following distributions: the Kumaraswamy Weibull $(\mathrm{Kw}-\mathrm{W})$ model with pdf given by

$$
f_{\mathrm{KW}-\mathrm{W}}(x)=a b \beta \alpha^{\beta} x^{\beta-1} \mathrm{e}^{-(\alpha \mathrm{x})^{\beta}}\left[1-\mathrm{e}^{-(\alpha \mathrm{x})^{\beta}}\right]^{\mathrm{a}-1}\left\{1-\left[1-\mathrm{e}^{-(\alpha \mathrm{x})^{\beta}}\right]^{\mathrm{a}}\right\}^{\mathrm{b}-1},
$$

the McDonald Weibull (McW) model with pdf given by

$$
\begin{aligned}
f_{\mathrm{McW}}(x)= & \frac{c \beta \alpha^{\beta}}{B(a, b)} x^{\beta-1} \exp \left\{-(\alpha x)^{\beta}\right\}\left[1-\exp \left\{-(\alpha x)^{\beta}\right\}\right]^{a c-1} \\
& \times\left\{1-\left[1-\exp \left\{-(\alpha x)^{\beta}\right\}\right]^{c}\right\}^{b-1}
\end{aligned}
$$

and the Libby-Novic beta Weibull (LNB-W) model with pdf given by

$$
f_{\mathrm{LNB}-\mathrm{W}}(x)=\frac{K \beta \alpha^{\beta} x^{\beta-1} \exp \left\{-(\alpha x)^{\beta}\right\}\left[1-\exp \left\{-(\alpha x)^{\beta}\right\}\right]^{a-1} \exp \left\{-(b-1)(\alpha x)^{\beta}\right\}}{\left\{1-(1-c)\left[1-\exp \left\{-(\alpha x)^{\beta}\right\}\right]\right\}^{a+b}},
$$

where $K=c^{a} / B(a, b), a>0, b>0, c>0, \alpha>0, \beta>0$ and $x>0$.

In Table 2, the MLEs and their standard errors (SEs) (in parentheses) of the parameters from nine fitted models and the Akaike Information Criterion (AIC), Consistent Akaike Information Criterion (CAIC) and Bayesian Information Criterion (BIC) values are presented. According to the lowest values of the AIC and CAIC statistics, the BMO-W model could be chosen as the best model among the nine fitted models. Formal tests for the extra shape parameters in the BMO-W distribution can be performed based on LR statistics. The results for comparing the models to the current data are given in Table 3 . The 
Table 2 MLEs (SEs in parentheses) for some fitted models to the failure time data and the AIC, CAIC and $\mathrm{BIC}$ values

\begin{tabular}{ccccccccc}
\hline Model & $a$ & $b$ & $c$ & $\alpha$ & $\beta$ & AlC & CAIC & BIC \\
\hline \multirow{2}{*}{ W } & - & - & - & 0.349 & 2.374 & \multirow{2}{*}{264.107} & 264.255 & 268.968 \\
& - & - & - & $(0.017)$ & $(0.210)$ & & & \\
EW & 0.284 & - & - & 0.253 & 5.747 & 261.211 & 261.511 & 268.504 \\
& $(0.054)$ & - & - & $(0.011)$ & $(0.693)$ & & & \\
BW & 0.274 & 0.785 & - & 0.266 & 5.864 & 263.167 & 263.673 & 272.890 \\
& $(0.042)$ & $(0.779)$ & - & $(0.056)$ & $(0.359)$ & & & \\
MOW & - & - & 27.588 & 1.247 & 1.058 & 262.491 & 262.791 & 269.783 \\
& - & - & $(57.410)$ & $(1.405)$ & $(0.514)$ & & & \\
EMOW & - & 0.280 & 0.838 & 0.249 & 5.920 & 263.160 & 263.667 & 272.883 \\
& - & $(0.046)$ & $(0.664)$ & $(0.017)$ & $(0.274)$ & & & \\
BMO-W & 0.262 & 0.259 & 0.086 & 0.293 & 7.450 & 260.389 & 261.159 & 272.543 \\
& $(0.046)$ & $(0.085)$ & $(0.080)$ & $(0.019)$ & $(0.014)$ & & & \\
\hline \multirow{2}{*}{ McW } & 3.551 & 0.108 & 0.091 & 0.715 & 2.875 & \multirow{2}{*}{264.212} & 264.981 & 276.366 \\
& $(1.140)$ & $(0.013)$ & $(0.008)$ & $(0.015)$ & $(0.017)$ & & & \\
\hline \multirow{2}{*}{ KW-W } & 0.266 & 1.336 & - & 0.231 & 6.515 & \multirow{2}{*}{262.948} & 263.454 & 272.671 \\
& $(0.048)$ & $(0.711)$ & - & $(0.032)$ & $(0.054)$ & & & \\
\hline \multirow{2}{*}{ LNB-W } & 0.281 & 0.287 & 7.281 & 0.298 & 6.807 & \multirow{2}{*}{261.158} & 261.928 & 273.313 \\
& $(0.048)$ & $(0.095)$ & $(6.055)$ & $(0.020)$ & $(0.066)$ & & & \\
\hline
\end{tabular}

rejection of the null models is significant for the five LR tests. So, we have evidence of the potential need for the three parameters of the BMO-W distribution for the current data.

The plots of the fitted BMO-W pdf and of the four fitted pdfs discussed before are displayed in Fig. 5. They indicate that the BMO-W distribution provides a better fit to these data compared to the other models. So, this distribution can be considered a very competitive model to the LNB-W distribution.

\subsection{Illustration 2: Plasma ferritin data}

Here, consider the data discussed by Weisberg (2014, Section 6.4) which represent 202 athletes collected at the Australian Institute of Sport. The variable evaluated in this study is the plasma ferritin concentration. These data were analyzed recently by (Cordeiro et al. 2014) using the Libby-Novic beta normal (LNB-N) distribution with density function given by

$$
f(x)=\frac{K \phi\left(\frac{x-\mu}{\sigma}\right)\left[\Phi\left(\frac{x-\mu}{\sigma}\right)\right]^{a-1}\left[1-\Phi\left(\frac{x-\mu}{\sigma}\right)\right]^{b-1}}{\sigma\left[1-(1-c) \Phi\left(\frac{x-\mu}{\sigma}\right)\right]^{a+b}},
$$

Table 3 LR tests

\begin{tabular}{cccc}
\hline Failures & Hypotheses & Statistic LR & $p$-value \\
\hline BMO-W vs W & $H_{0}: a=b=c=1$ vs $H_{1}: H_{0}$ is false & 9.717 & 0.010 \\
BMO-W vs EW & $H_{0}: b=c=1$ vs $H_{1}: H_{0}$ is false & 4.822 & 0.045 \\
BMO-W vs BW & $H_{0}: c=1$ vs $H_{1}: H_{0}$ is false & 4.777 & 0.017 \\
BMO-W vs MOW & $H_{0}: a=b=1$ vs $H_{1}: H_{0}$ is false & 6.101 & 0.024 \\
BMO-W vs EMOW & $H_{0}: a=1$ vs $H_{1}: H_{0}$ is false & 4.771 & 0.017 \\
\hline
\end{tabular}




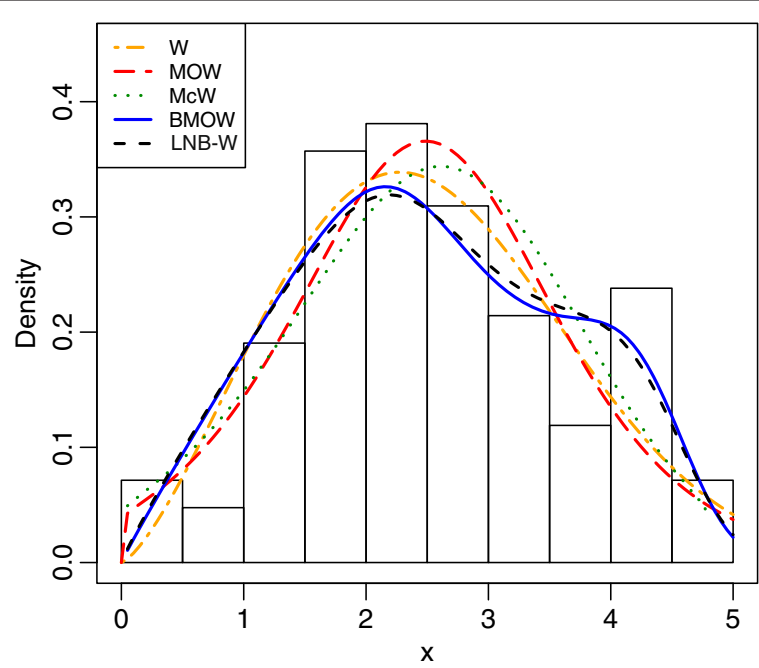

Fig. 5 Fitted densities for the failure data

where $x \in R, \mu \in R$ is a location parameter, $\sigma>0$ is scale parameter, $a, b$ and $c$ are positive shape parameters and $\phi(\cdot)$ and $\Phi(\cdot)$ are the pdf and cdf of the standard normal distribution, respectively.

In Table 4, the MLEs and their SEs (in parentheses) of the parameters from fitted nine models and the AIC, CAIC and BIC values are presented. According to the lowest values of these statistics, the BMO-N model could be chosen as the best model among the nine fitted models. Formal tests for the extra shape parameters in the BMO-N distribution can be performed based on LR statistics. The results for comparing the models to the current data are given in Table 5. The rejection of the null models is significant for the five LR

Table 4 MLEs (SEs in parentheses) for some fitted models to the failure time data and the AIC, CAIC and $\mathrm{BIC}$ values

\begin{tabular}{ccccccccc}
\hline Model & $a$ & $b$ & $c$ & $\mu$ & $\sigma$ & AIC & CAIC & BIC \\
\hline \multirow{2}{*}{ N } & - & - & - & 76.887 & 47.399 & 2135.994 & 2136.054 & 2142.611 \\
& - & - & - & $(3.335)$ & $(2.359)$ & & & \\
EN & 329.601 & - & - & -242.079 & 109.357 & 2088.197 & 2088.318 & 2098.122 \\
& $(257.441)$ & - & - & $(50.515)$ & $(9.321)$ & & & \\
BN & 7.349 & 0.185 & - & -12.984 & 30.265 & 2083.599 & 2083.803 & 2096.833 \\
& $(1.252)$ & $(0.014)$ & - & $(0.003)$ & $(0.003)$ & & & \\
MON & - & - & 0.040 & 158.760 & 51.373 & 2090.927 & 2091.048 & 2100.851 \\
& - & - & $(0.023)$ & $(18.248)$ & $(3.962)$ & & & \\
EMON & - & 18.598 & 0.007 & 124.403 & 58.756 & 2068.369 & 2068.572 & 2081.602 \\
& - & $(10.644)$ & $(0.003)$ & $(17.340)$ & $(3.442)$ & & & \\
BMO-N & 6.373 & 0.542 & 0.007 & 111.698 & 41.750 & 2065.363 & 2065.669 & 2081.904 \\
& $(6.622)$ & $(0.161)$ & $(0.003)$ & $(15.687)$ & $(7.625)$ & & & \\
\hline \multirow{2}{*}{ MCN } & 0.156 & 0.182 & 25.619 & 17.890 & 28.867 & \multirow{2}{*}{2074.582} & 2074.888 & 2091.123 \\
& $(0.013)$ & $(0.016)$ & $(0.165)$ & $(0.357)$ & $(0.254)$ & & & \\
\hline \multirow{2}{*}{ KW-N } & 2.980 & 0.229 & - & 12.806 & 29.581 & \multirow{2}{*}{2099.027} & 2099.230 & 2112.260 \\
& $(0.073)$ & $(0.016)$ & - & $(0.197)$ & $(0.042)$ & & & \\
\hline \multirow{2}{*}{ LNB-N } & 2.661 & 0.520 & 58.246 & 108.256 & 38.987 & \multirow{2}{*}{2067.884} & 2068.190 & 2084.425 \\
& $(1.659)$ & $(0.139)$ & $(26.941)$ & $(13.437)$ & $(6.660)$ & & & \\
\hline
\end{tabular}


Table $5 \mathrm{LR}$ tests

\begin{tabular}{cccc}
\hline Plasma & Hypotheses & Statistic LR & $p$-value \\
\hline BMO-N vs N & $H_{0}: a=b=c=1$ vs $H_{1}: H_{0}$ is false & 76.631 & $<0.001$ \\
BMO-N vs EN & $H_{0}: b=c=1$ vs $H_{1}: H_{0}$ is false & 26.834 & $<0.001$ \\
BMO-N vs BN & $H_{0}: c=1$ vs $H_{1}: H_{0}$ is false & 20.236 & $<0.001$ \\
BMO-N vs MON & $H_{0}: a=b=1$ vs $H_{1}: H_{0}$ is false & 29.564 & $<0.001$ \\
BMO-N vs EMON & $H_{0}: a=1$ vs $H_{1}: H_{0}$ is false & 5.005 & 0.015 \\
\hline
\end{tabular}

tests. So, we have a clear evidence for the three parameters of the BMO-N distribution when modeling data of this type. The plot of the fitted BMO-N pdf and the four fitted pdfs discussed before are displayed in Fig. 6. They indicate that the BMO-N distribution provides the best fit to these data compared to the other models. Finally, the proposed distribution can be considered a very competitive model to the LNB-N distribution.

\section{Concluding remarks}

We define a new class of models, named the beta Marshall-Olkin-G (BMO-G) family of distributions by adding three shape parameters, which generalizes some well-known distributions in the statistical literature such as the normal, Weibull and beta distributions. We provide a mathematical treatment of the proposed family including expansions for the density function, ordinary and incomplete moments and generating function. The BMO$\mathrm{G}$ density function can be expressed as a mixture of exponentiated density functions. This property is important to obtain several other results. We derive a power series for the quantile function. Our formulas related to the BMO-G model are manageable, and with the use of modern computer resources with analytic and numerical capabilities, they may turn into adequate tools for applied statisticians. Some special models are explored. The estimation of the model parameters is carried out by the method of maximum likelihood. Finally, we fit some special models in the new family to two real data sets to demonstrate their potentiality.

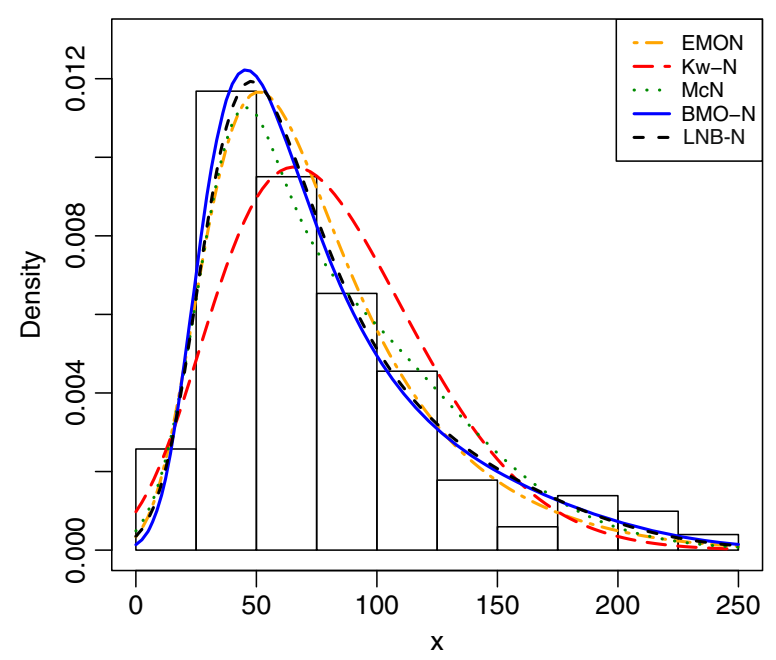

Fig. 6 Fitted densities for the plasma data 


\section{Endnote}

\section{${ }^{1}$ http://functions.wolfram.com/06.23.06.0004.01}

\section{Competing interests}

The authors declare that they have no competing interests.

\section{Authors' contributions}

MA developed the mathematics of paper and participated in drafting the manuscript. EB developed Sections 3 and 13, produced article figures and participated in drafting the manuscript. GMC and CGBD were advisors and reviewed all the work from the initial idea, through preparation of the manuscript until the final version. All authors read and approved the final manuscript.

\section{Author details}

${ }^{1}$ Department of Statistics, Persian Gulf University of Bushehr, Bushehr, Iran. ${ }^{2}$ Departamento de Estatística, Universidade Federal de Pernambuco, Recife, Pernambuco, Brazil. ${ }^{3}$ Departamento de Estatística, Universidade Federal da Bahia, Salvador, Bahia, Brazil. ${ }^{4}$ Departamento de Ciências Exatas, Universidade de São Paulo, Piracicaba, São Paulo, Brazil.

Received: 6 January 2015 Accepted: 10 June 2015

Published online: 01 July 2015

\section{References}

Alexander, C, Cordeiro, GM, Ortega, EMM, Sarabia, JM: Generalized beta-generated distributions. Comput. Stat. Data Anal. 56, 1880-1897 (2012)

Alzaatreh, A, Lee, C, Famoye, F: A new method for generating families of continuous distributions. METRON. 71, 63-79 (2013)

Bourguignon, M, Silva, RB, Cordeiro, GM: The Weibull-G family of probability distributions. J. Data Sci. 12, 53-68 (2014)

Cordeiro, GM, Alizadeh, M, Ortega, EMM: The exponentiated half-logistic family of distributions: Properties and applications. J. Probab. Stat. 2014, 1-21 (2014)

Cordeiro, GM, Castro, M: A new family of generalized distributions. J. Stat. Comput. Simul. 81, 883-898 (2011)

Cordeiro, GM, Nadarajah, S: Closed-form expressions for moments of a class of beta generalized distributions. Braz. J. Probab. Stat. 25, 14-33 (2011)

Cox, DR, Hinkley, DV: Theoretical statistics. Chapman Hill, London (1979)

Doornik, J: Ox 5: An Object-Oriented Matrix Language. Timberlake Consultants Press, London (2007)

Eugene, N, Lee, C, Famoye, F: Beta-normal distribution and its applications. Commun. Stat. Theory Methods. 31, 497-512 (2002)

Exton, H: Handbook of Hypergeometric Integrals: Theory, Applications, Tables, Computer Programs. Halsted Press, New York (1978)

Gupta, RC, Gupta, PL, Gupta, RD: Modeling failure time data by Lehman alternatives. Commun. Stat. Theory Methods. 27, 887-904 (1998)

Gupta, RC, Gupta, RD: Proportional reversed hazard rate model and its applications. J. Stat. Planning Inference. 137, 3525-3536 (2007)

Jayakumar, K, Mathew, T: On a generalization to marshall-olkin scheme and its application to burr type xii distribution Stat. Papers. 49, 421-439 (2008)

Jones, MC: Families of distributions arising from distributions of order statistics. Test. 13, 1-43 (2004)

Kenney, JF, Keeping, ES: Mathematics of Statistics, Part 1. 3rd edition, Van Nostrand, New Jersey (1962)

Marshall, AW, Olkin, I: A new method for adding a parameter to a family of distributions with application to the exponential and Weibull families. Biometrika. 84, 641-652 (1997)

Moors, JJA: A quantile alternative for kurtosis. The Statistician. 37, 25-32 (1988)

Murthy, DNP, Xie, M, Jiang, R: Weibull models. New Jersey (2004)

R Development Core Team: R: A Language and Environment for Statistical Computing. R Foundation for Statistical Computing, Viennas, Austria (2013)

Rényi, A: On measures of entropy and information, volume I. In: proceedings of the 4th Berkeley symposium on mathematical statistics and probability edition. University of California Press, Berkeley, (1961)

Rigby, RA, Stasinopoulos, DM: Generalized additive models for location, scale and shape (with discussion). Appl. Stat. 54, 507-554 (2005)

Shannon, CE: Prediction and entropy of printed english. Bell Syst. Tech. J. 30, 50-64 (1951)

Trott, M: The Mathematica Guidebook for Symbolics. With 1 DVD-ROM (Windows, Macintosh and UNIX). Springer, New York (2006)

Weisberg, S: Applied linear regression. 3rd edition. Wiley, New York (2014)

Zografos, K, Balakrishnan, N: On families of beta- and generalized gamma-generated distributions and associated inference. Stat. Methodol. 6, 344-362 (2009) 